\title{
Scenario Based Two-Stage Stochastic Programming Approach for the Midterm Production Planning of Oil Refinery
}

\author{
${ }^{1}$ Norshela Mohd Noh, ${ }^{2}$ Arifah Bahar* and ${ }^{3}$ Zaitul Marlizawati Zainuddin \\ ${ }^{1,2,3}$ Department of Mathematical Sciences, Faculty of Science \\ Universiti Teknologi Malaysia, 81310 Johor Bahru, Johor, Malaysia \\ ${ }_{1,2,3}$ UTM Centre for Industrial and Applied Mathematics, \\ Universiti Teknologi Malaysia, 81310 Johor Bahru, Johor, Malaysia \\ *Corresponding author: arifah@utm.my
}

Article history

Received: 9 September 2018

Received in revised form: 5 December 2018

Accepted: 19 December 2018

Published on line: 31 December 2018

\begin{abstract}
Recently, oil refining industry is facing with lower profit margin due to uncertainty. This causes oil refinery to include stochastic optimization in making a decision to maximize the profit. In the past, deterministic linear programming approach is widely used in oil refinery optimization problems. However, due to volatility and unpredictability of oil prices in the past ten years, deterministic model might not be able to predict the reality of the situation as it does not take into account the uncertainties thus, leads to non-optimal solution. Therefore, this study will develop two-stage stochastic linear programming for the midterm production planning of oil refinery to handle oil price volatility. Geometric Brownian motion (GBM) is used to describe uncertainties in crude oil price, petroleum product prices, and demand for petroleum products. This model generates the future realization of the price and demands with scenario tree based on the statistical specification of GBM using method of moment as input to the stochastic programming. The model developed in this paper was tested for Malaysia oil refinery data. The result of stochastic approach indicates that the model gives better prediction of profit margin.
\end{abstract}

Keywords Two-stage stochastic programming; geometric Brownian motion; scenario based approach; oil refinery optimization; production planning

Mathematics Subject Classification 97K60, 90C15.

\section{Introduction}

Oil refinery production need to make important decisions such as to determine the right amount of crude oil to purchase, the products to produce and to optimize the production with the best use of the existing resources in order to improve the company's profitability. In the past, all these decisions do not incorporate uncertainty where the deterministic linear programming approach is widely used in oil refinery optimization problems. In the year 2008, the crude oil prices rose to the maximum price and fell to a minimum price in the same year. The drastic 
price changes also affect the petroleum finish product prices as well as demand. Due to volatile and unpredictable oil price over the past ten years, the deterministic model might not be able to predict the real situation as it does not take into account the uncertainties that lead to a non-optimal solution. Thus, this study will develop two-stage stochastic programming for the midterm production planning of oil refinery to represent the problem using a scenariobased approach to handle uncertainties such as prices and demand of finish products. This approach attempts to maximize profit of oil refinery midterm production planning subjected to uncertainties which is using the geometric Brownian motion (GBM) model. Accurately incorporating uncertainty into the refinery production planning problem will result in better decision making and improve refinery expected profit and will lead to increase in country's GDP and government's revenue. A real case study based on Malaysia oil refinery data was used to test the developed model

The rest of this paper is organized as follows. In the next section, we will present a literature review for the stochastic optimization of oil refinery. In section 3, we first model and forecast crude oil price, the price of finish products and demand of finish products following GBM. Then we discretize continuous probability distribution of forecast values to generate discrete scenario with binomial scenario tree based on the statistical specification of GBM using first (expected mean) and second (variance) moment matching method as input to the two-stage stochastic programming. Then in section 4 and 5 we will discuss the results and conclusions.

\section{Literature Review}

Leiras et al. [1] made a review for oil refineries planning under uncertainties papers from the 90s to 2010 and only 5 out of 22 papers studied the two-stage stochastic programming. Khor et al. [2] studied hybrid two-stage stochastic programming and employed sampling method to construct scenarios for the price, demand of finish products and yield uncertainty. Other studies also developed two-stage stochastic programming approach with financial risk management for the real case study of Bangchak Petroleum Public Company Limited production planning Pongsakdi et al. [3] and found that stochastic model gives higher profit with lower risk where the number of scenarios for the uncertainties constructed via sampling. The latest paper for the two-stage model reviewed by Leiras et al. [1] was Ribas et al. [4] paper where the scenarios construction for uncertainties is based on basic scenario (low-25\%, base-50\%, high-25\%) and they compared two-stage stochastic programming with robust min-max regret model and max-min model. However, this basic scenario is not appropriate to represent actual possible realization for each source uncertainty due to inconsideration of volatility in actual data. Therefore, Awudu and Zhang [5] modelled the uncertainties (prices of end products) following the GBM to solve two-stage stochastic programming for biofuel supply chain. Chung and Yang [6] also modelled the demand of products as GBM in proposing a scenario based two-stage stochastic programming model for the control or dummy wafers problem. In this study, simplified representation of formulation for the deterministic linear program is based on the study by Khor [7] and we develop a scenario based two-stage stochastic programming where GBM model is used to represent uncertainties. Therefore, moment matching method is used to construct a decision tree for oil price and demand uncertainty as input to the stochastic programming to maximize the expected profit of midterm production planning of oil refinery. Real data of prices and demand of Tapis crude oil, gasoline, naphtha, kerosene, diesel, fuel oil from 1990 to 2014 were 
used in this study and was tested for the oil refinery production planning.

\section{Model Formulation}

\subsection{Deterministic Model for Oil Refinery Midterm Production P lanning}

In the deterministic model, crude oil price, finish products sales price and demand of finish products are constant. The mean values from historical data are used. The objective function is to maximize midterm production planning of oil refinery considered as revenue from products sales minus raw material cost and operating cost. This study is close to Khoret al.[2] study where the objective function and constraints are presented below

Maximize profit $Z$

$$
=\sum\left[\begin{array}{l}
\sum_{i \in I} \gamma_{i, t} S_{i, t}+\sum_{i \in I} \tilde{\gamma}_{i, t} I_{i, t}^{f}-\sum_{i \in I} \lambda_{i, t} P_{i, t}-\sum_{i \in I} \tilde{\lambda}_{i, t} I_{i, t}^{S}-\sum_{j \in J} C_{j, t} x_{j, t}-\sum_{i \in I} h_{i, t} H_{i, t}- \\
\sum_{j \in J}\left(\alpha_{j, t} C E_{j, t}+\beta_{j, t} y_{j, t}\right)-\left(r_{t} R_{t}+o_{t} O_{t}\right)
\end{array}\right]
$$

subject to

a) Production capacity constraints

$$
\begin{gathered}
x_{j, t}=x_{j, t-1}+C E_{j, t} \forall j \in J, \\
y_{j, t} C E_{j, t}^{L} \leq C E_{j, t} \leq y_{j t} C E_{j, t}^{U} \forall j \in J \forall t \in T,
\end{gathered}
$$

where

$$
y_{j, t}= \begin{cases}1 & \text { if there is an expansion } \\ 0 & \text { otherwise }\end{cases}
$$

b) Demand constraints

$$
\begin{gathered}
S_{i, t}+L_{i, t}=d_{i, t} \forall i \in I^{\prime}, \\
d_{i, t}^{L} \leq S_{i, t} \leq d_{i, t, s}^{U} \forall i \in I^{\prime} .
\end{gathered}
$$

c) Availability constraints

$$
p_{t}^{L} \leq P_{t} \leq p_{t}^{U} \forall i \in I^{\prime}
$$

d) Inventory requirements

$$
\begin{gathered}
I_{i, t}^{f \min } \leq I_{i, t}^{f} \leq I_{i, t}^{f \max } \forall i \in I^{\prime}, \\
I_{i, t}^{f}=I_{i, t+1}^{S} \forall i \in I^{\prime} .
\end{gathered}
$$

e) Material balances

$$
P_{t}+I_{i, t}^{S}+\sum_{j \in J} b_{i, j} x_{j, t}-S_{i, t}-I_{i, t}^{f}=0 \forall i \in I^{\prime}, \forall t \in T .
$$

The simplified oil refinery operation based on Khoret et al. [2] study is used to describe a formulation of the deterministic linear program for midterm production planning as in Figure 1.

Where the decision variables in unit barrel per year are $x_{1}$ refer to the mass flow rate of crude oil stream, $x_{2}$ mass flow rate of gasoline, $x_{3}$ mass flow rate of naphta, $x_{4}$ mass flow rate of 
jet fuel or kerosene, $x_{5}$ mass flow rate of heating oil or diesel, $x_{6}$ mass flow rate of fuel oil and $x_{14}$ mass flow rate of cracker feed stream required within objective function. These represent the crude oil being purchased and finish products being sold while the other variables are present in constraints.

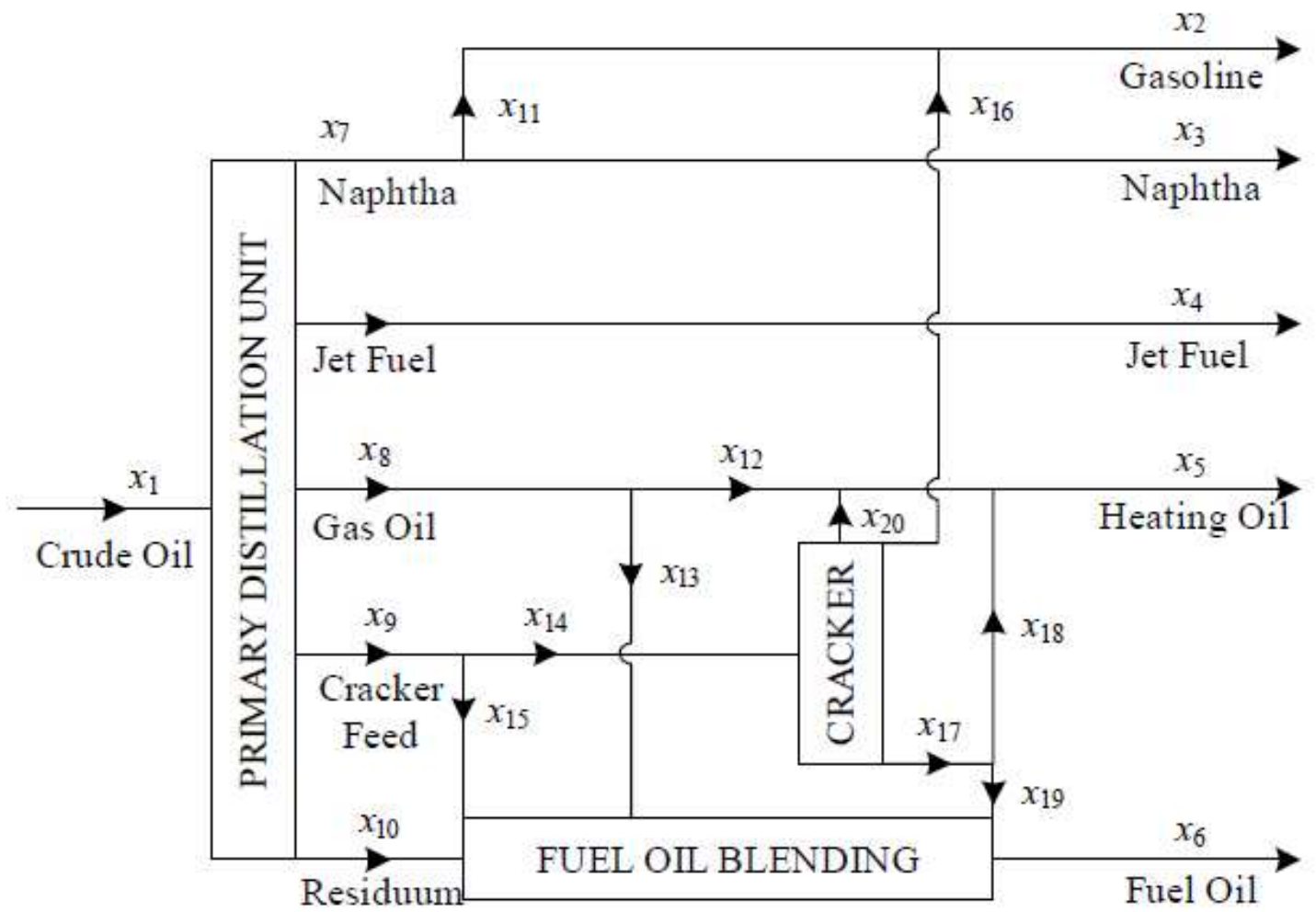

Figure 1: Simplified Oil Refinery Midterm Production Planning for Formulation of Deterministic Linear Model

\subsection{Stochastic Model for Oil Refinery Midterm Production Planning}

The general formulation for two-stage stochastic linear programming problem is

$$
\begin{gathered}
\max C^{T} x+E[Q(x, \zeta)] \\
\text { s.t } A x \leq b, x \geq 0
\end{gathered}
$$

where $Q(x, \zeta)$ is the optimal value for the second stage problem,

$$
\begin{gathered}
\max q^{t} y \\
\text { s.tT } x+W y \leq h, y \geq 0
\end{gathered}
$$

The objective function in (11) contains first stage decisions (before the realization of uncertainty) denoted by vector $x$ and the second stage decisions (after the realization of uncertainty) denoted by $y$. Matrix $A$, vector $b$ and vector $C$ are known with certainty. In the second stage 
problem, elements $q T W$ and $h$ are viewed as random and in this study we only consider element $q$ and $h$ as random due to prices and demand of finish products uncertainty.

The amount of crude oil purchased, amount of finish products sold and production capacity is the first stage decision variables. After the prices and demand for finish products uncertainty are revealed, the recourse costs are imposed based on corrective action. These corrective actions are associated with amount of unsatisfied demand and excess finish products. The recourse costs are denoted as penalty cost incurred due to shortfall and surpluses in oil refinery production planning due to the uncertainty of finish products demand. A scenario based approach in this study refer to the random space is considered as discrete events. The discrete distribution with finite number of $s$ possible outcomes (scenarios) $\zeta_{s}=\left(q_{s} T_{s}, W_{s} h_{s}\right)$ corresponds to the probability of $p_{s}$. Hence equation (11) and (12) can be written as large linear programming model

$$
\begin{gathered}
\max C^{T} x+\sum_{s=1}^{S} p_{s} q_{s}^{T} y_{s} \\
\text { s.t } A x=b \\
T_{s} x+W_{s} y_{s}=h_{s}, s=1, \ldots, S \\
x \geq 0, y_{s} \geq 0, s=1, \ldots, S
\end{gathered}
$$

\subsection{Stochastic Parameters Model and Scenario Tree Construction}

In the year 2008, crude oil prices rose to the maximum price and fell to a minimum in the same year. The impact of drastic price changes also affects the petroleum finish product prices as well as demand. Instability of crude oil price attracted researches to find the best model to describe the behavior of crude oil price Postali et al. [8], [9] and [10]. Based on that, we consider that the random variables, the prices and demand of finish products are modelled with GBM

$$
d S_{t}=\mu S_{t} d t+\sigma S_{t} d W_{t}
$$

where $S$ is the prices and demands, $\mu$ and $\sigma$ are the drift and volatility and $W$ is a Brownian motion. Sample path of Brownian motion $W$ are not differentiable so the equation (14) has the explicit solution

$$
S_{t}=S_{0} \exp \left[\left(\mu-\frac{\sigma^{2}}{2}\right) t+\sigma W_{t}\right]
$$

GBM has a property that the log ratio follows normal distribution

$$
\ln \left(\frac{S_{\mathbf{t}}}{S_{\mathbf{t}-1}}\right) \sim N\left(\left[\mu-\frac{\sigma^{2}}{2}\right] t, \sigma^{2} t\right)
$$

It can be shown that GBM has a Markov process which the future independent of past, given the present.

In order to generate discrete number of scenarios based on GBM, Jarrow and Rudd [11] proposed modelis used and the first (drift) and second moment (variance) of binomial steps are matching with the GBM. The next upward and downward price and probability nodes are calculated using the measurements as follow. 
Price at the next upward node:

$$
S_{x_{i}}^{t}=S_{x_{i}}^{1} \exp \left(\left[\mu_{x_{i}}-\frac{\sigma_{x_{i}}^{2}}{2}\right] t+\sigma_{x_{i}}^{2} \sqrt{t}\right)
$$

with probability

$$
p=0.5
$$

Price at the next downward node:

$$
S_{x_{i}}^{t}=S_{x_{i}}^{1} \exp \left(\left[\mu_{x_{i}}-\frac{\sigma_{x_{i}}^{2}}{2}\right] t-\sigma_{x_{i}}^{2} \sqrt{t}\right)
$$

with probability

$$
1-p=0.5
$$

$\mu$ and $\sigma$ are calculated using maximum likelihood estimation.

\section{Results}

\subsection{Stochastic Model}

A new reformulated objective function consists of summation of maximization of the expected profit (considered as revenue from products sales minus raw material cost and operating cost) and minimization of summation of expected recourse penalty due to shortfall and surpluses in production.

$$
\operatorname{Max} Z_{1}=E[Z]-E_{s, \text { demand }}
$$

Since the objective function in deterministic model is linear, the expectation objective function with random price coefficient is given by

$$
E[Z]=\sum_{i \in I} \sum_{s \in S} p_{\mathbf{s}} C_{i} x_{i}, i=\{1,2,3,4,5,6,14\} \in I_{\text {price }}^{\text {random }} \subseteq I, s=\{1,2,3\} \in S
$$

where $p_{s}$ is the probability of scenario $s$ for prices uncertainty, $C_{i}$ and $x_{i}$ are vector consisting of cost coefficients and production flow rate of products type $i$. The corresponding expected recourse penalty due to uncertainties in demand for the second stage cost is given by

$$
E_{s, \text { demand }}=\sum_{i \in I} \sum_{s \in S} p_{s}\left(c_{i}^{+} z_{i, s}^{+}+c_{i}^{-} z_{i, s}^{-}\right), i=\{2,3,4,5,6\} \in I \subseteq I, s=\{1,2,3\} \in S
$$

where $p_{s}$ is the probability of scenario $s$ for demand uncertainty, $z_{i, s}^{+}$and $z_{i, s}^{-}$are an amount of under production and overproduction of product type $i$ per realization of scenario $s$ and $c_{i}^{+}$and $c_{i}^{-}$are penalty costs for shortfall and surplus in production of product type $i$. Subject to first stage constraints; limitation on plant capacity, mass balances (fixed yields, fixed blends and unrestricted balances) and second stage constraints; demand constraints. In the second stage constraints, the demand deterministic constraints are replaced by the new constraints to model the number of generated scenarios in the stochastic model. Thus the new demand constraints are

$$
x_{i}+\mathbf{z}_{i, s}^{+}-\mathbf{z}_{i, s}^{-}=d_{i, s}, i=\{2,3,4,5,6\} \in I_{\text {demand }}^{\text {random }} \subseteq I, s=\{1,2,3\} \in S
$$


where $d_{i, s}$ is demand of finish products $i$ in scenario $s$. In this study, the formulation for twostage stochastic programming is close to Khor et al. [2] study however we model the uncertain parameters as GBM.

\subsection{Scenario Tree}

In this study, we consider seven uncertain parameters for prices uncertainty and five parameters for finish products demand uncertainty. Each parameter takes on two values, high value which is denoted as $\mathrm{H}$ and low value which is denoted as $\mathrm{L}$ as shown in Table 1 and Table 2. The probability of each occurring high and low value is 0.5 . We obtain 128 scenarios $\left(2^{7}\right)$ with the probability of occurrence of each scenario is 0.0078 by multiplying the probabilities of uncertain parameter in each scenario as presented in the scenario tree in Figure 2. For example, in scenario 1, $\{\mathrm{H}, \mathrm{H}, \mathrm{H}, \mathrm{H}, \mathrm{H}, \mathrm{H}, \mathrm{H}\}$ is a set of event sequences denotes as high prices for Tapis, Gasoline, Naphta, Kerosene, Diesel, Fuel oil and Cracker feed. While for demand scenario tree, 32 scenarios $\left(2^{5}\right)$ were constructed with the probability of occurrence of each scenario is 0.03125 as presented in Figure 3.

Table 1: Simplified Oil Refinery Midterm Production Planning for Formulation of Deterministic Linear Model

\begin{tabular}{|l|c|c|}
\hline Item & High price $\mathbf{( \$ / \text { barrel) }}$ & Low price $\mathbf{( \$ / \text { barrel) }}$ \\
\hline Tapis Crude Oil & 115.76 & 104.07 \\
\hline Gasoline & 128.01 & 106.57 \\
\hline Naphta & 111.55 & 88.18 \\
\hline Kerosene & 127.34 & 110.15 \\
\hline Diesel & 131.86 & 107.72 \\
\hline Fuel Oil & 103.41 & 74.24 \\
\hline Cracker feed & 15 & 5 \\
\hline
\end{tabular}

Table 2: High and Low Values for the Uncertain Prices

\begin{tabular}{|c|c|c|}
\hline Item & High demand (barrel/year) & Low demand (barrel/year) \\
\hline Gasoline & 97348862 & 95682387 \\
\hline Naphta & 160747 & 140042 \\
\hline Kerosene & 160747 & 140042 \\
\hline Diesel & 75519186 & 74764408 \\
\hline Fuel Oil & 1863729 & 1489348 \\
\hline
\end{tabular}

First and second stage programming model can be summed up to the large linear programming model by taking all possible scenarios for prices and all scenarios for demand uncertainty. The objective function equation is extended to large scale linear programming model and there 


\section{Scenario tree structure}

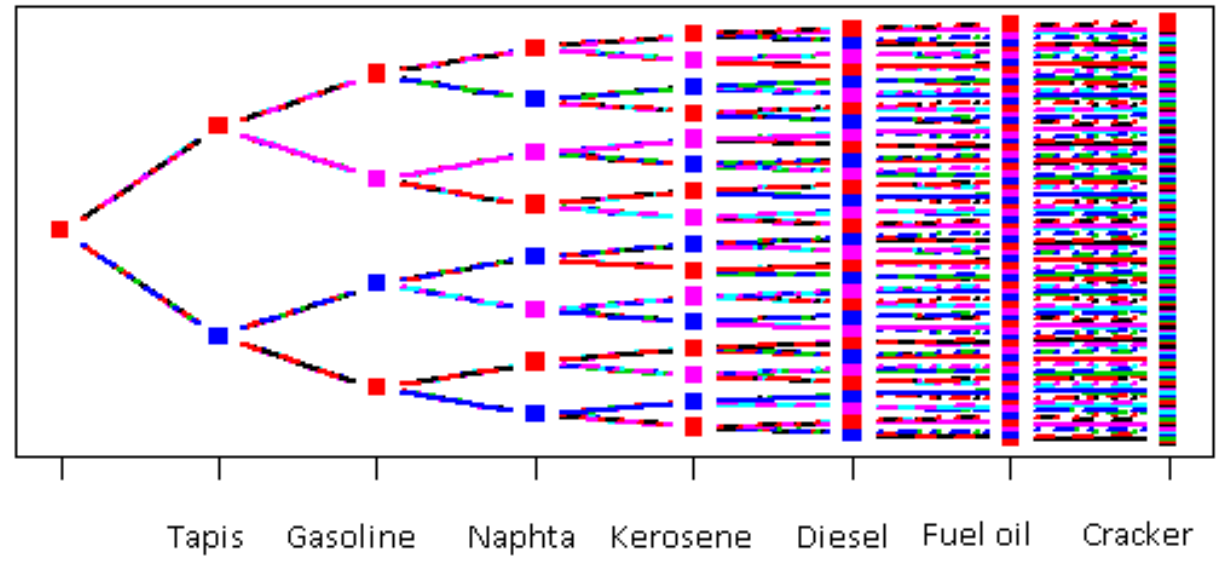

Scenario 1

Scenario 2

Scenario 3

Scenario 128

Figure 2: High and Low Values for the Uncertain Demands

\section{Scenario tree structure}

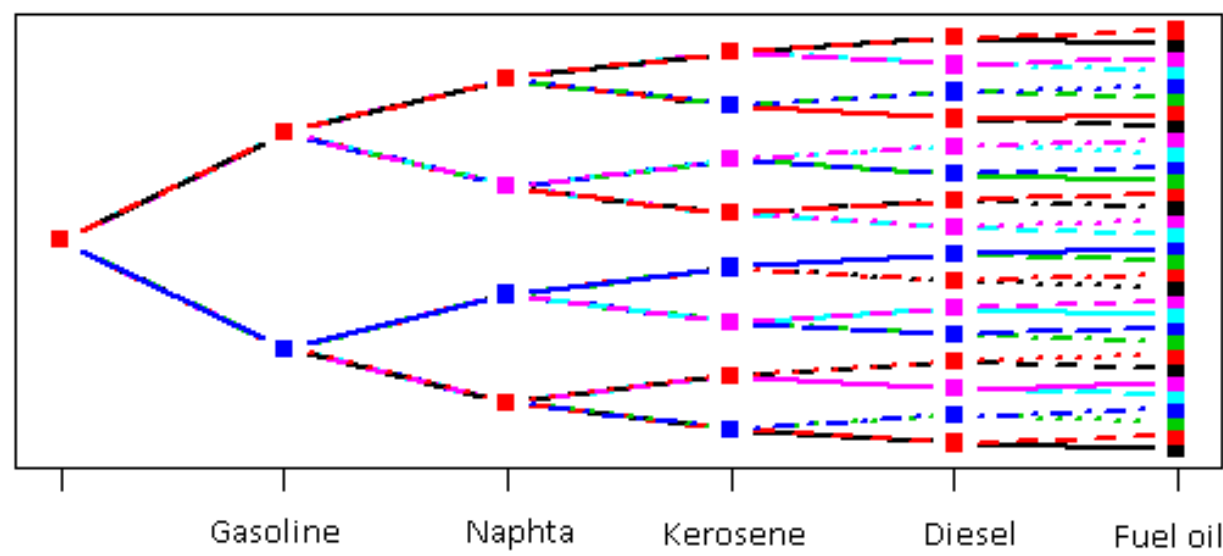

Scenario 1

Scenario 2

Scenario 3

Scenario 31

Scenario 32

Figure 3: Scenario Tree for Prices Uncertainty 
are 160 new constraints for demand uncertainty. $\mathrm{R}$ was used to generate scenarios and construct the scenario tree. Meanwhile, the deterministic and stochastic programming model was implemented using GAMS.

We found that stochastic model gives higher expected profit compared to deterministic model as in Table 3.

Table 3: Scenario Tree for Demand of Finish Products Uncertainty

\begin{tabular}{|c|c|c|}
\hline Model & Deterministic (\$/year) & Stochastic $(\$ /$ year) \\
\hline Profit & 24655770 & 26762972 \\
\hline
\end{tabular}

In this study, value of stochastic solution (VSS) is used to evaluate the stochastic model.

$$
V S S=T S P-E E V
$$

Where TSP refer to the objective value of two-stage stochastic programming model and $E E V$ refer to expected solution of deterministic model. Therefore, the stochastic model gained $8.54 \%$ more than deterministic model.The result indicates that the stochastic model gives better prediction of oil refinery midterm production planning profit margin.

\section{Conclusions}

This paper presents a study to maximize the profit of midterm production planning of oil refinery profit with price and demand uncertainty. Each price and demand follows the GBM. Hence, we generate the future realization of the price and demands with scenario tree based on the statistical specification of GBM using method of moment. A scenario-based two-stage stochastic programming with recourse was developed and test with Malaysian oil refinery data. The result shows that the stochastic model gives higher expected profit compared to the deterministic model. It indicates that the stochastic model gives a better prediction of oil refinery midterm production planning profit margin.

\section{Acknowledgement}

The author would like to thank the Ministry of High Education (MOHE), GE STEM Grant with vote no. A. J091002.5600.07397 for the financial support, R.J130000.7809.4F440 and Q.J130000.2426.03G54 for the partial financial support, Universiti Teknologi Malaysia.

\section{References}

[1] Leiras, A., Ribas, G., Hamacher, S. and Elkamel, A. Literature review of oil refineries planning under uncertainty. Int. J. Oil, Gas Coal Technol. 2011. 4(2): 156-173,

[2] Khor, C. S., Elkamel, A., Ponnambalam, K. and Douglas, P. L. Two-stage stochastic programming with fixed recourse via scenario planning with economic and operational 
risk management for petroleum refinery planning under uncertainty. Chem. Eng. Process. Process Intensif. 2008. 47(9-10): 1744-1764.

[3] Pongsakdi, A., Rangsunvigit, P., Siemanond, K. and Bagajewicz, M. J. Financial risk management in the planning of refinery operations. Int. J. Prod. Econ. 2006. 103(1): 6486 ,

[4] Ribas, G. P., Hamacher, S. and Street, A. Optimization under uncertainty of the integrated oil supply chain using stochastic and robust programming. Int. Trans. Oper. Res. 2010. 17(6): 777-796.

[5] Awudu, I. and Zhang, J. Stochastic production planning for a biofuel supply chain under demand and price uncertainties. Appl. Energy. 2013. 103: 189-196.

[6] Chung, S. H. and Yang, Y. S. A scenario-based stochastic programming model for the control or dummy wafers downgrading problem. Appl. Stoch. Model. Bus. Ind. 2009. 25: 263-274.

[7] Khor, C. S. A Hybrid of stochastic programming approaches with economic and operational risk management for petroleum refinery planning under uncertainty. 2006.

[8] Postali, F. A. S. and Picchetti, P. Geometric Brownian motion and structural breaks in oil prices: a quantitative analysis. Energy Econ. 2006. 28(4): 506-522.

[9] Mostafei, H., Sani, A. A. R. and Askari, S. A methodology for the choice of the best fitting continuous-time stochastic models of crude oil price. Int. J. Energy Econ. Policy. 2013. 2: $137-142$.

[10] Mirkhani, S. and Saboohi, Y. Stochastic modeling of the energy supply system with uncertain fuel price - a case of emerging technologies for distributed power generation. Appl. Energy. 2012. 93: 668-674.

[11] Jarrow, R. and Rudd, A. Approximate option valuation for arbitrary stochastic processes. J. Financ. Econ. 1982. 10(3): 347-369.

\section{Appendix}

\section{Set and Indices}

$\begin{array}{ll}I^{\prime} & \text { set of material or products } i \\ J & \text { set of processes } j \\ S & \text { set of scenarios } s\end{array}$

\section{First-stage Decision Variable}

$P_{t} \quad$ amount of crude oil purchase in period $t$

$x_{j, t}$

$S_{i, t}$ production capacity of process $j$ during period $t$

$L_{i, t}$ amount of product $i$ sold in period $t$

$H_{i, t}$ amount of lost demand for product $i$ in period $t$ amountof product $i$ to be outsourced in period $t$ 


\section{Recourse Parameters}

$c_{i}^{+} \quad$ fixed penalty cost per unit demand $d_{i, s}$ of under production product $i$ per realization of scenario $s$

$c_{i}^{-} \quad$ fixed penalty cost per unit demand $d_{i, s}$ of under production product $i$ per realization of scenario $s$

\section{Deterministic Parameters}

$b_{i, j} \quad$ stoichiometric coefficient for material $i$ in process $j$

$C_{j, t} \quad$ operating cost of process $j$ in period $t$

$d_{i, t, s} d_{i, t, s}^{L} d_{i, t, s}^{U}$ demand for product $i$ in period $t$ per realization of scenario $s$ with its corresponding constant lower and upper bounds

$I_{i, t}^{f \min } I_{i, t}^{f \max }$ minimum and maximum required amount of inventory for material $i$ at the end of period $t$

$h_{i, t} \quad$ unit cost of outsourcing the production of product type $i$ in period $t$

$P_{t} \quad$ amount of crude oil purchase in period $t$

$p_{t}^{L} p_{t}^{U} \quad$ lower and upper bounds of the availability of crude oil during period $t$

$r_{t} O_{t} \quad$ labour cost for per man-hour of regular and overtime in period $t$

$\alpha_{j, t} \quad$ cost coefficient for the investment cost of capacity expansion of process $j$ in period $t$

$\beta_{j, t} \quad$ fixed cost charge for capacity expansion investment cost of process $j$ in period $t$

$\gamma_{i, t} \quad$ unit sales price of product type $i$ in period $t$

$\tilde{\gamma}_{i, t} \quad$ value of the final inventory of material $i$ in period $t$

$\lambda_{i, t} \quad$ unit purchase price of crude oil type $i$ in period $t$

$\check{\lambda}_{i, t} \quad$ value of the starting inventory of material $i$ in period $t$

\section{Stochastic Parameters}

$p_{s} \quad$ probability of scenario $s$

$\lambda_{t, s} \quad$ unit purchase price of crude oil in period t per realization of scenario $s$

$\gamma_{i, s, t} \quad$ unit sales price of product type $i$ in period $t$ per realization of scenario $s$

$d_{i, s, t} \quad$ demand for product $i$ in period $t$ per realization of scenario $s$

\section{Second-stage Decision Variables}

$z_{i, s}^{+}$

amount of underproduction of product type $i$ per realization of scenario $s$ amount of overproduction of product type $i$ per realization of scenario $s$ 